\title{
Avoiding impression tears in gingival embrasures of anterior preparations
}

\section{Michele M. Harutunian, Angela De Bartolo, Denise Estafan}

Cariology and Comprehensive care Department, New York University College of Dentistry, New York, USA

Email:. mmh10@nyu.edu, adb2005@nyu.edu, de1@nyu.edu

Received 6 July 2013; revised 4 August 2013; accepted 28 August 2013

Copyright (c) 2013 Michele M. Harutunian et al. This is an open access article distributed under the Creative Commons Attribution License, which permits unrestricted use, distribution, and reproduction in any medium, provided the original work is properly cited.

\begin{abstract}
As restorative dentists, we are faced everyday with decisions about which impression materials and techniques should be used when restoring teeth. The challenge becomes bigger when the patients present with open gingival embrasures, the dreaded "black triangles", which tops the list of dentist frustration while taking the impression and after preparation of veneers, crowns, bridges, etc. The technique described offers an option to avoid problems related to impressions locking in gingival embrasures and tearing on removal.
\end{abstract}

Keywords: Black Triangles; Impression Tears; Gingival Embrasures; Polyvinyl Siloxane Impression Material

\section{INTRODUCTION}

Polyvinyl siloxane impression materials have excellent accuracy, stability and tear strength for fixed restorations. While sufficient tear strength is needed for full coverage restorations, particularly when margins are sub-gingival, it can complicate impression taking with partial coverage restorations in patients with periodontal attachment loss [1].

When preparing porcelain veneers, if the proximal contact is left intact, gingival recession related to periodontal disease creates a space between the proximal contact and the gingival papilla. This space is known as the "black triangle space". Impression material can flow into that space and tear as it is removed from the mouth [2-5], (Figure 1). The resulting impression is either insufficient (pieces missing, margins missing) or inaccurate (distorted from tearing). The clinician must further prepare the teeth, open the proximal contact or change the impression technique.

Extending the preparation to obliterate the proximal contact negates the problem of impression tearing, but it can also reduce the success of the porcelain veneer. Less enamel may be available for bonding and the margin location may end up in an occlusal contact area [6].

Changing the impression technique may also create challenges. The black triangle spaces are adjacent to the margins of the veneer preparations [7]. Block-out materials (petroleum jelly, cotton) can potentially interfere with capturing the margins in an impression-unless the block-out is created with the same material as the impression itself.

A technique is presented below for avoiding impression tears around veneer preparations in area of gingival recession.

Impression making remains a challenging procedure due to the potential for voids and tears, which may adversely affect the precise fabrication of indirect restorations.

Thin sections of impression materials are susceptible to tearing in gingival crevices and interproximal spaces.

Polyvinyl siloxanes materials are currently considered

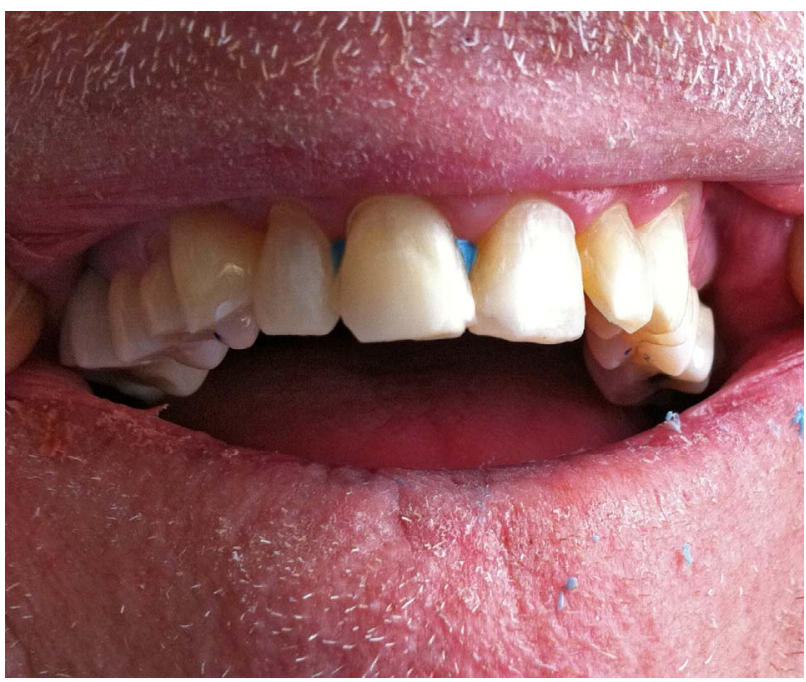

Figure1. Impression material torn after removal. 
to reproduce the greatest detail of all the impression materials, preparing electronic versions of their papers.

\section{TECHNIQUE}

A healthy 56-year-old male who had gone five years without visiting a dentist presented to New York University College of Dentistry for dental treatment. A comprehensive examination was performed and he was treatment planned to have porcelain veneers and crowns to enhance his smile. It was noted from the start that he presented with existing black triangles due to his mild periodontal disease.

Following discussions of risks and benefits of the treatment and obtaining informed consent, 4 teeth were prepared for porcelain veneers and 1 full ceramic crown.

Medium body polyvinyl siloxane material (Reprosil, Denstply Caulk, Milford, DE) was used. The tray fit was checked and loaded. This material was left to set in place according to the manufacturer's instructions, 3 minutes working time and 3 minutes setting time. Once the second phase of impression material set, the tray was removed. The result was a torn impression in the cervical areas of the teeth, (black triangles), shown in (Figure 1).

Medium body polyvinyl siloxane material (Reprosil, Denstply Caulk, Milford, DE) was placed at the palatal of the prepared teeth, short of the margins (Figure 2). It was left to set in place according to the manufacturer's instructions. The tray was removed. The impression material exhibited a "controlled tear" away from the preparation and margins (Figure 3). The junction of the 2 phases of polyvinyl siloxane provides a convenient place for the impression to tear.

Medium body polyvinyl siloxane material (Reprosil, Dentsply Caulk, Milford, DE) was placed at the palatal

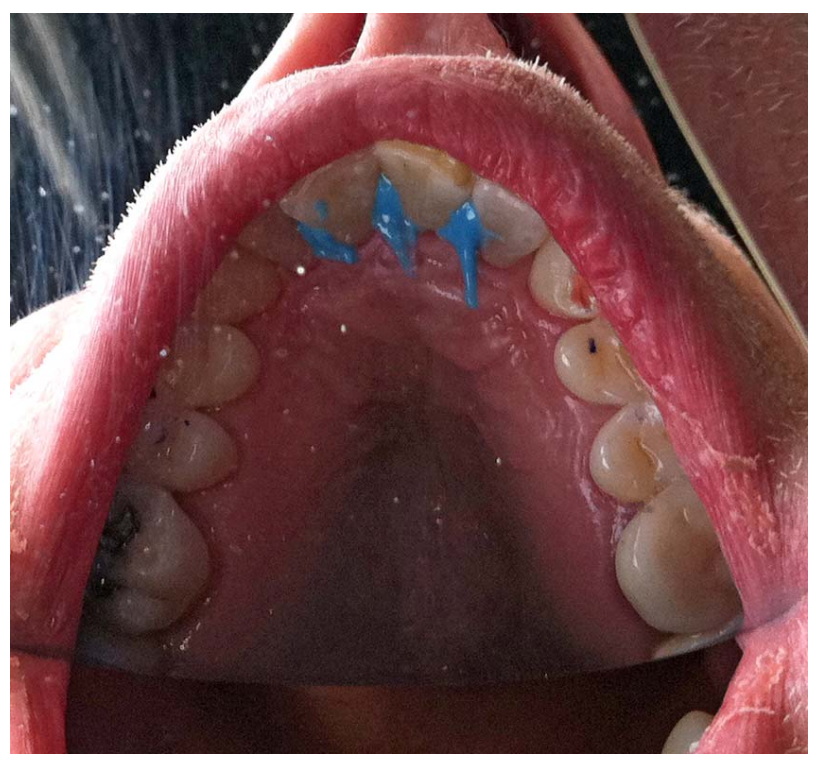

Figure 2. Palatal impression material placement.

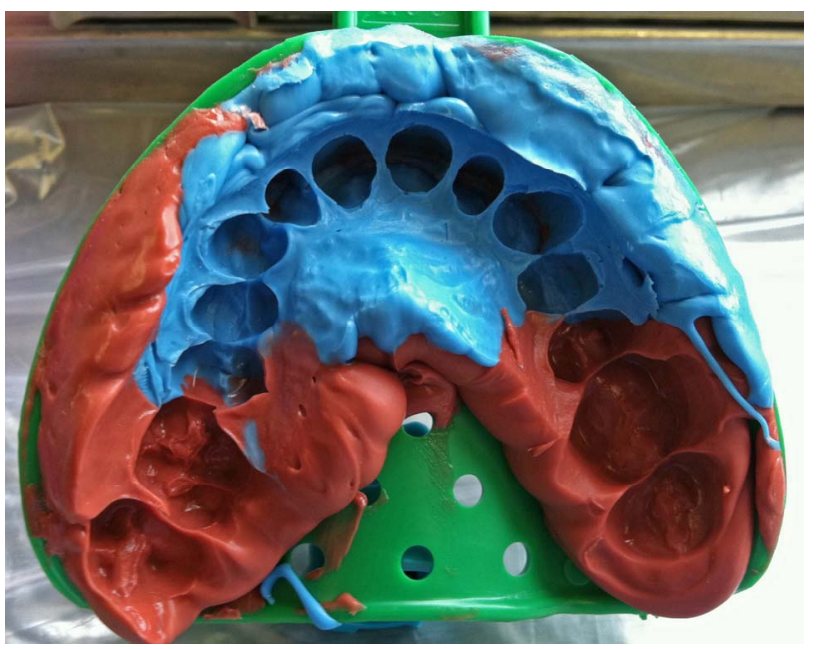

Figure 3. “Controlled tear” impression after removal.

of the prepared teeth, short of the margins (Figure 2). This material was left to set in place according to the manufacturer's instructions, 3 minutes working time and 3 minutes setting time. The tray fit was checked and loaded with the same impression material. Once the second phase of impression material set, the tray was removed. The impression material exhibited a "controlled tear" away from the preparation and margins (Figure 3). The junction of the 2 phases of polyvinyl siloxane provides a convenient place for the impression to tear.

\section{DISCUSSION}

Dealing with black triangles is one of the most challenging esthetic problems. One third of adults have unaesthetic black triangles, which are more appropriately referred to as open gingival embrasures. Besides being unsightly and prematurely aging the smile, black triangles are prone to accumulation of food debris and excessive plaque.

Polyvinyl siloxanes are currently considered to reproduce the greatest detail of all the impression materials. [1-3]. They are inherently hydrophobic but new "hydrophilic" polyvinyl siloxanes have been introduced with the manufacturer claims that they better wet moist dental surfaces [7].

The technique described in our clinical case allowed for a new lesson learned: realizing that polyvinyl siloxane materials exhibit hydrophilic properties, we were able to combine the new material with the existing material and achieve a favorable impression eliminating the "black triangles" in the cervical area where the material was torn.

With advances in innovative materials and their properties, new and improved clinical techniques are developed to elevate patient care. This technique offers an option to avoid problems related to impression locking in 
gingival embrasures and tearing on removal.

\section{ACKNOWLEDGEMENTS}

Dr. Harutunian and Dr. De Bartolo extend a special "thank you" to their mentor and co-author, Dr. Denise Estafan for her dedication to teaching cosmetic dentistry in an enjoyable, hands-on environment.

\section{REFERENCES}

[1] Hondrum, S.O. (1994) Tears and energy properties of three impression materials. The International Journal of Prosthodontics, 7, 517-521.

[2] Pant, R., Juszczyk, A.S., Clark RK and Radford, D.R. (2008) Long-term dimensional stability and reproduction of surface detail of four polyvinyl siloxane duplicating materials. Journal of Dentistry, 36, 456-461. http://dx.doi.org/10.1016/j.jdent.2008.03.003

[3] Hartmann, R. and Muller, F. (2004) Clinical studies on the appearance of natural anterior teeth in young and old adults. Gerodontology, 21, 10-16. http://dx.doi.org/10.1111/j.1741-2358.2004.00009.X

[4] Li, J.H., DI, P., Hu, X.L., Qiu, L.X., Cui, H.Y. and Lin, Y. (2010) Clinical study of the application of non-invasive porcelain veneer to improve the aesthetic effects in maxillary anterior area. Beijing Da Xue Xue Bao, 42, 103-107

[5] Cutbirth, S.T. (1992) Restoration of maxillary anterior teeth using porcelain jacket crowns and porcelain veneers. Journal of Esthetic Dentistry, 4, 1-5. http://dx.doi.org/10.1111/j.1708-8240.1992.tb00645.x

[6] Silva, N.R., Bonfante, E.A., Martins, L.M., Valverde, G.B., Thompso, V.P., Ferencz, J.L. and Coelho, P.G. (2012) Reliability of reduced-thickness and thinly veneered lithium disilicate crowns. Journal of Dental Research, 91, 305-310.

[7] Hamalian, T.A., Nasr, E. and Chidiac, J.J. (2011) Impression materials in fixed prosthodontics: Influence on choice on clinical procedure. Journal of Prosthodontics, 20, 153-160. 